\title{
A Summary of Recent Experimental Research on Ion Energy and Charge States of Pulsed Vacuum Arcs
}

\author{
E.M. Oks ${ }^{1,2^{*}}$, G.Yu. Yushkov ${ }^{2}$ and A. Anders ${ }^{3}$ \\ ${ }^{1}$ State University of Control Systems and Radioelectronics, Tomsk, 634050, Russia. \\ ${ }^{2}$ High Current Electronics Institute, Russian Academy of Sciences, Tomsk 634055, Russia. \\ ${ }^{3}$ Lawrence Berkeley National Laboratory, Berkeley, California 94720, USA
}

\begin{abstract}
The paper reviews the results of vacuum arc experimental investigations made collaboratively by research groups from Berkeley and Tomsk over the last two years, i.e. since the last ISDEIV in 2006. Vacuum arc plasma of various metals was produced in pulses of a few hundred microseconds duration, and the research focussed on three topics: (i) the energy distribution functions for different ion charge states, (ii) the temporal development of the ion charge state distribution, and (iii) the evolution of the mean directed ion velocities during plasma expansion.

A combined quadruple mass-to-charge and energy analyzer (EQP by HIDEN Ltd) and a time-of-flight spectrometer were employed. Cross-checking data by those complimentary techniques helped to avoid possible pitfalls in interpretation. It was found that the ion energy distribution functions in the plasma were independent of the ion charge state, which implies that the energy distribution on a substrate are not equal to due to acceleration in the substrate's sheath. In pulsed arc mode, the individual ion charge states fractions showed changes leading to a decrease of the mean charge state toward a steady-state value. This decrease can be reduced by lower arc current, higher pulse repetition rate and reduced length of the discharge gap. It was also found that the directed ion velocity slightly decreased as the plasma expanded into vacuum.
\end{abstract}

\section{INTRODUCTION}

In spite of more then 100 years of studies and applications of vacuum arcs with cathodic spot phenomena, this kind of discharge remains both an important and interesting subject for further investigations. It is known that all physical processes responsible for ionization, emission mechanism, current transfer and others occur in a small region near the cathode surface. The plasma composition becomes approximately "frozen" in further plasma expansion to the anode. That fact gives the possibility to investigate the cathode processes of vacuum arcs based on the analysis and characteristics of the parameters of the plasma far away from the cathode. Therefore, the parameters of ion beams extracted from vacuum arc plasma include information about cathode processes. Thus the combination of a vacuum arc ion source with a timeof-flight mass/charge spectrometer or other ion beam analysis system is an attractive experimental tool to investigate the basic processes of the vacuum arc. This approach to experimental research of vacuum arc discharge operation has been used in the collaborative work between the Berkeley and Tomsk groups for over 10 years. Our common discharge system is usually fed by a pulse forming network giving a pulse duration of a few hundred microseconds. Previously, we have investigated ion charge state distributions, ion directed velocities, ion erosion rates of cathode materials, fast Fourier transform (FFT) analysis of arc discharge noise, and some other properties. Results of these investigating were reported in the Proceedings of the ISDEIV [1] and elsewhere [26].

This paper summarizes the results of vacuum arc experimental investigations made collaboratively since the last ISDEIV in 2006. Three main subjects were investigated recently: (i) the energy distribution functions for different ion charge states, (ii) the temporal development of ion charge state distributions, and (iii) the evolution of the mean ion directed velocities during plasma expansion.

\section{ENERGY DISTRIBUTION FUNCTIONS FOR DIFFERENT ION CHARGE STATES}

To investigate ion energy distribution functions, a combined quadruple mass/charge and energy analyzer (EQP by HIDEN Ltd) was used. The cathodic arc plasma was streaming towards the grounded entrance orifice of the differentially pumped analyzer, which was placed about $45 \mathrm{~cm}$ away from the cathode. The analyzer operated in ion mode, i.e. without auxiliary ionization. Data acquisition was synchronized with the pulsed arc by using the analyzer's external gating capability. The data acquisition was done over the entire arc pulse duration. To reduce the influence of the large plasma fluctuations ("noise"), which is common for vacuum arcs, each measurement was repeated five times, and in some experiments even ten times, giving us sets of raw data distributions. These sets were used to calculate the average distribution functions with exhibited much reduced noise compared to the raw data functions. The analyzer's raw data are acquired as energy per charge, and therefore, 
when considering multiply charged ions, the raw data need to be multiplied by the charge state number to obtain the correct energy distribution function. The energy range is therefore $0-100 \mathrm{eV}$ for singly charged ions and $0-200$ $\mathrm{eV}$ for the doubly charged. Additionally, an electron emitting probe was used to determine the plasma potential, which is needed to account for sheath effects at the analyzer's sampling orifice.

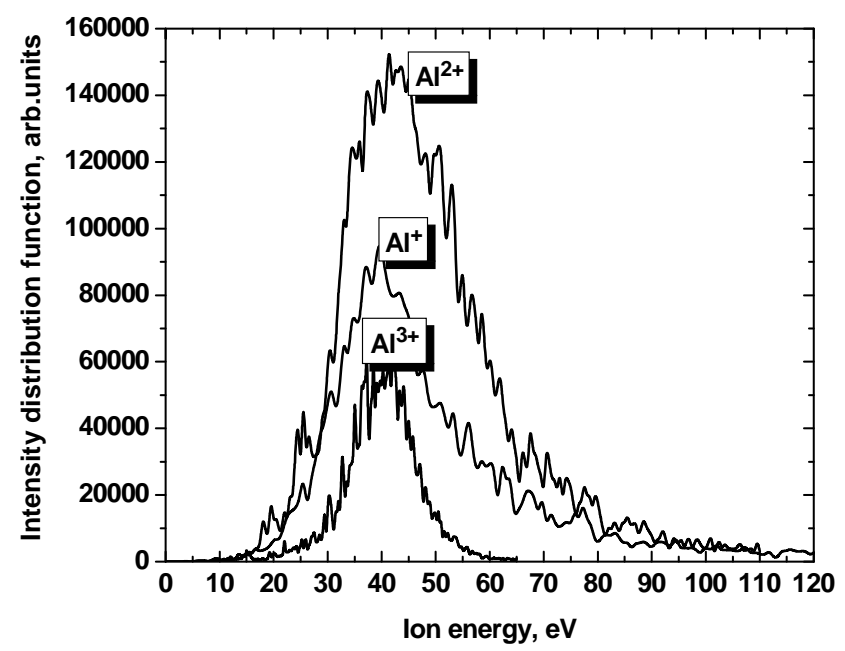

Fig.1. Energy distribution functions in the plasma for an aluminum cathodic arc, with current $200 \mathrm{~A}, 350 \mu$ s, 1 p.p.s.

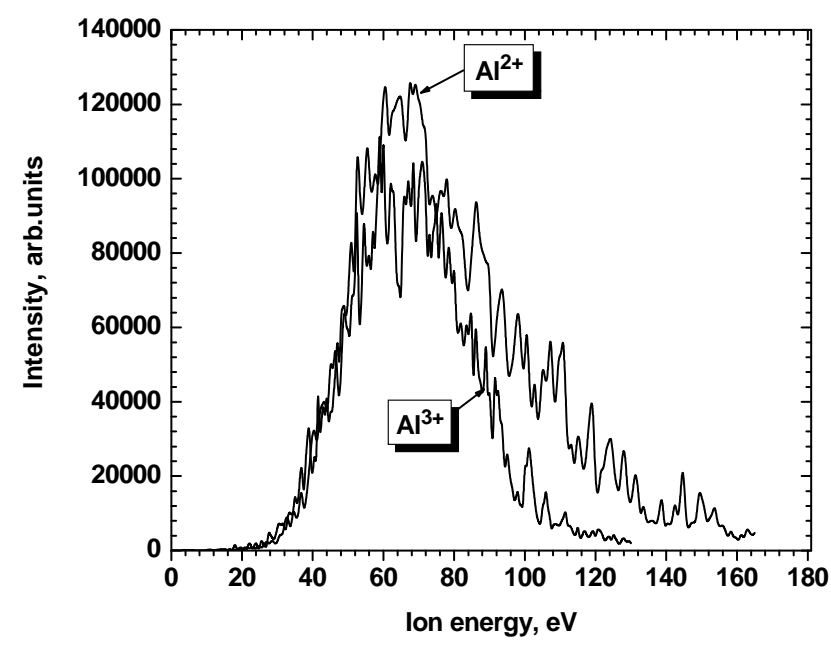

Fig.2. Energy distribution functions in the plasma for aluminum arc with a magnetic field of $1.4 \mathrm{kG}$; arc current $200 \mathrm{~A}, 350 \mu \mathrm{s}, 1$ p.p.s.

The plasma potential was found to be slightly negative in our system, which required a small correction of the analyzer's raw data to account for a charge-statedependent deceleration of ions in the sheath at the analyzer's entrance. The corrected data indicated that, for a given cathode material, the most likely ion energy in the plasma is independent of the charge state. Fig. 1 presents as an example the results for aluminum arcs. Those energy analyzer results are is in good agreement with previous results obtained by time-of-flight (TOF) measurements [6]. One should note that equal ion energies in the plasma imply different ion energies for different charge states when arriving on a substrate or detector unless such substrate or detector is precisely at the plasma potential.

The magnetic field is known to play an important role for the enhancement of the ion energy, and here it is shown that its effect is similar for the different charge states (Fig.2). By distinguishing between kinetic and potential energies of ions, the findings can be consolidated with other literature data. Experiments with various short arc durations confirmed that the ion energy is enhanced at the beginning of an arc; the energy distribution functions are initially broader and extend much further into the high energy range.

More detailed description of experimental facility and measurement procedure as well as other experimental results of ion energy spectra can be found elsewhere [7].

\section{TEMPORAL DEVELOPMENT OF THE ION CHARGE STATE DISTRIBUTIONS}

It is known that the mean charge state of ions in vacuum arc plasmas is between 1 and 4, depending on cathode material, arc current, arc pulse duration, presence or absence of magnetic field at the cathode, as well background gas pressure. A characteristic of the vacuum arc is a significant decrease of the mean ion charge state throughout the pulse: this decrease can be observed up to a few milliseconds, until the "noisy" steady-state value of a DC arc is established.

The experiments were done with the vacuum arc metal ion source "Mevva-V" at Berkeley Laboratory. Different metals ( $\mathrm{Al}, \mathrm{Mg}, \mathrm{Cu}, \mathrm{Pb}, \mathrm{Bi}, \mathrm{Mo}$ ) were used as cathode in the experiments. The charge state distributions were measured at selected delay times after arc triggering using the time-of-flight (TOF) spectrometer. The TOF instrument has a drift length of $1.03 \mathrm{~m}$ and the detector was a magnetically suppressed Faraday cup. Each arc discharge of typically $300 \mathrm{~A}$ had a duration of $300 \mu \mathrm{s}$, determined by the pulse-forming network, at a repetition rate of up to from 0.1 to 10 pulses per second (p.p.s.). A high repetition rate ensured that, under base pressure conditions of about $10^{-4} \mathrm{~Pa}$, a gas layer cannot form on the cathode surface between arc pulses. The effects of gas and metal vapor were studied by (i) introducing nitrogen gas into the cathode region; and (ii) by intentionally introducing additional surfaces with which the expanding metal plasma can interact. Upon introduction of gas near the cathode, the pressure enhancement was essentially limited to the region between cathode and extraction grid system because the extraction grid system 
acted as an array of flow-limiting orifices to the main vacuum chamber where the cryogenic pump was located, i.e. we are dealing with a differentially pump TOF spectrometer.

In experiments with different distances between cathode and anode, the standard 18-cathode holder was removed and a movable, single-cathode plasma source installed. The cathode size was the same as with the 18cathode source, namely a rod of $1 / 4$ inch $(6.25 \mathrm{~mm})$ diameter. As usual for our experiments, the cathode was inserted into an alumina sleeve so that the arc spots can only burn on the rod's front surface.

For all cathode materials investigated, the arc current, the pulse repetition rate, and the length of the cathodeanode distance have an essential influence on the development of the charge state distribution. Despite constant discharge parameters, the ion beam mean charge state showed the familiar, significant decrease during each arc pulse. It was shown that for a given pulse duration, the mean ion charge state decreased more at lower arc current, low pulse repetition rate, and when the distance between cathode and anode was increased. The observed effects may be associated with charge exchange processes in the discharge plasma.

The experimental data of the decrease of $\bar{Q}$ can be fitted with a first-order exponential function of the form

$$
\bar{Q}(t)=A \exp (-t / \tau)+\bar{Q}_{S S},
$$

where $A$ is a factor that describes the relevance of the decay, $\tau$ is the characteristic decay time, and $\bar{Q}_{s s}$ is the steady-state average charge state number which is approached for $t>>\tau$. The strongest decay is seen with $\mathrm{Bi}$, which could be explained by the very low thermal conductivity in conjunction with a high vapor pressure of this material. These results indicate an important role of neutrals (both metal and gas) for charge exchange processes in the discharge volume between cathode and anode and beyond.

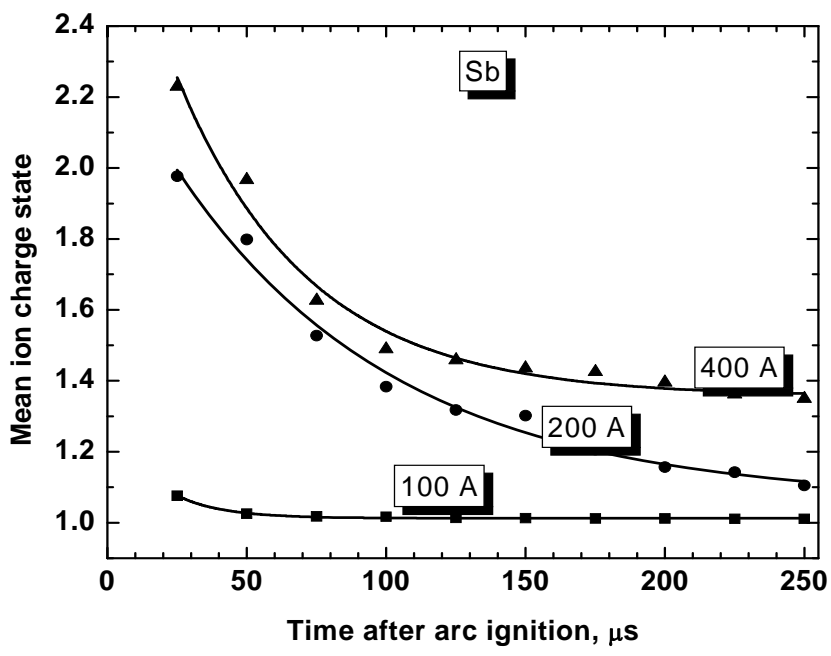

Fig.3. Temporal developments of ion mean charge state for antimony cathode and anode-cathode distance of $30 \mathrm{~cm}$, with arc pulse $350 \mu$ s, 1p.p.s.
For some applications it is desirable to have a pure, single charge state of ions. Based on the experiments of temporal development we realized that one way to decrease the ion charge is to increase the cathode-anode distance such as to promote charge exchange collisions. As an example, Fig. 3 present results with an antimony (Sb) cathode. One can see that there are only single charged ions in the plasma when using a low arc current and considering the time after the first $50 \mu \mathrm{s}$ of the arc pulse at $30 \mathrm{~cm}$ from cathode.

A more detailed description of the experimental facilities and measurement procedures as well as other experimental results on the temporal development of ion charge states in vacuum arc plasmas can be found elsewhere [8-11].

\section{MEASUREMENTS OF THE DIRECTED ION VELOCITIES}

During the investigations of the influence of the cathodeanode distance on the temporal development of the ion charge states it was also observed that there is a clear delay between arc starting and the appearance of the ion beam current (Fig.4). The ion current is extracted through a grid system located at a given distance from the cathode. Taking into account this delay time vs. the cathode-grid distance, it is possible to determine the most likely directed ion velocities at the beginning of the pulse. From Fig. 4 we obtained $v_{i}=0.43 * 10^{4} \mathrm{~m} / \mathrm{s}$ for bismuth (Bi) ions. For comparison, the value for titanium (Ti) ions was $v_{i}=2.1 * 10^{4} \mathrm{~m} / \mathrm{s}$. Those values are in very good agreement with our previous experiments [6].

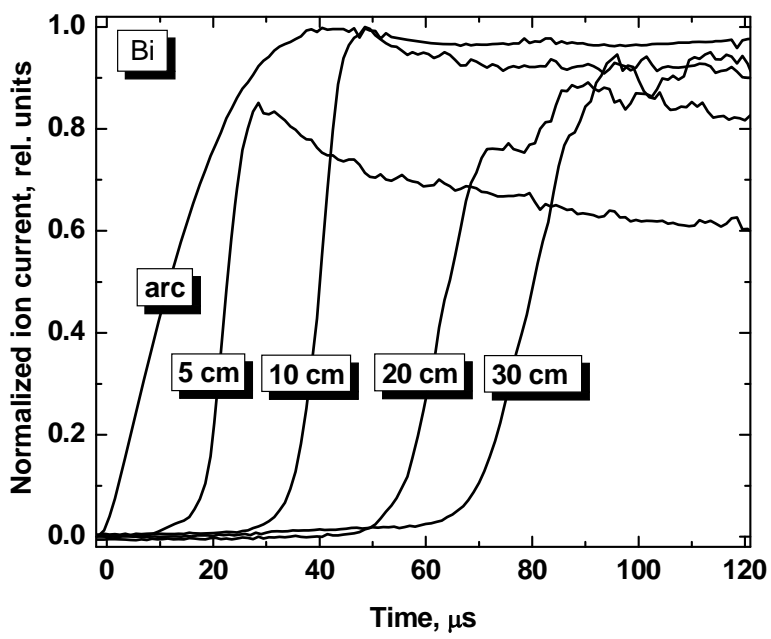

Fig.4. Time delays between arc current and ion beam extracted from anode located at different distances from the Bi-cathode, arc current $100 \mathrm{~A}, 350 \mu \mathrm{s}, 1$ p.p.s.

The next experimental step was to measure the development of the directed ion velocity as the plasma 
expanded. To measure the local velocities, 6 ion current probes were placed along a line at different distances from the cathode. In these experiments, the ion velocities were measured using the so-called current spike method, i.e., the arc current was modulated by a single short current spike or "jump" and we measured how fast this current perturbation propagates with the expanding plasma. Based on the delay between the current "jump" and a spike of the probe current it was possible to estimate the ion directed velocities. It was found that the velocities are roughly constant although there was a slight tendency to decrease during plasma expansion (Fig.5). An increase of arc current or introduction of a magnetic field lead to higher velocities but the distributions remained about the same when measuring for different distances from the cathode.

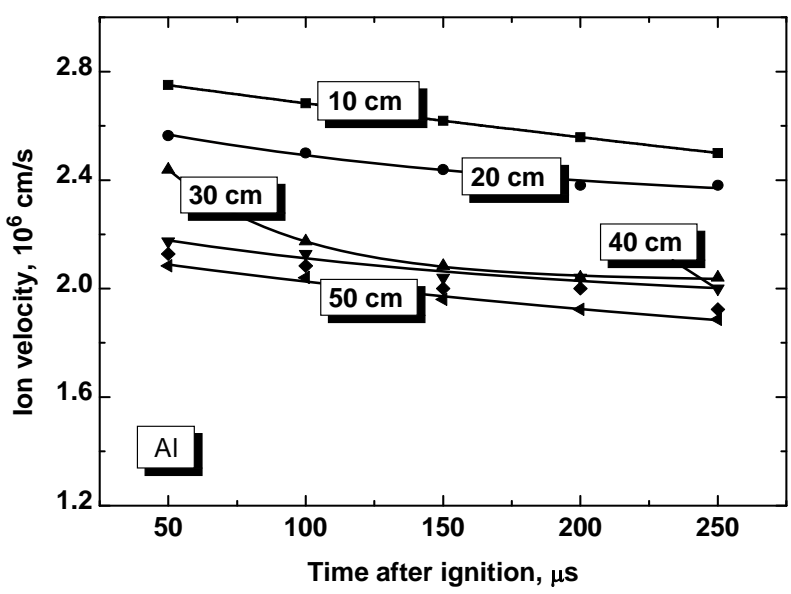

Fig.5. Temporal development of the ion direct velocities at different distances from the $\mathrm{Al}$ cathode, arc current $200 \mathrm{~A}, 350$ $\mu$ s, 1p.p.s.

\section{ACKNOWLEGMENTS}

The authors are grateful to Dr. Ian Brown (Berkeley Lab) for his continued interest and fruitful discussions. The work is supported by the US Department of Energy, IPP program, project LBNL-T2-0196, and under DOE contract AC02-05CH11231.

\section{REFERENCES}

[1] See papers of E.M. Oks, G.Yu.Yushkov, A Anders, and other co-authors in previous ISDEIV Proceedings (1996-2006)

[2] E.M Oks, A. Anders, I.G. Brown, M.R. Dickinson and R.A. MacGill, Ion charge state distributions in high current vacuum arc plasmas in a magnetic field, IEEE Trans. Plasma Sci., 1996, vol. 24, pp. 1174-1183.

[3] G.Yu.Yushkov, E.M. Oks, A. Anders and I.G Brown, Effect of multiple current spikes on the enhancement of ion charge states of vacuum arc plasmas, J. Appl. Phys., 2000, vol.87, pp. $8345-8350$.

[4] A. Anders, E.M. Oks and G.Yu Yushkov, Cathodic arcs: frac- tal voltage and cohesive energy rule, Appl. Phys. Lett., 2005, vol.86, pp. 211503-1-3.

[5] A. Anders, E.M. Oks, G.Yu Yushkov, K.P. Savkin, I.G. Brown and A.G. Nikolaev, Measurements of the total ion flux from vacuum arc cathode spots, IEEE Trans. Plasma Sci., 2005, vol.33, pp. 1532-1536.

[6] G.Yu. Yushkov, E.M. Oks, A. Anders and I.G Brown, Ion velocities in vacuum arc plasmas, J. Appl. Phys., 2000, vol.88, pp. 5618-5622.

[7] E.M. Oks and A. Anders, Charge-state-resolved ion energy distribution functions of cathodic vacuum arcs: A study involving the plasma potential and biased plasmas, J. Appl. Phys, 2007, vol. 102, pp. 043304/1-6.

[8] G. Yu. Yushkov and A. Anders, Cathodic Vacuum Arc Plasma of Thallium, IEEE Trans. on Plasma Sci., 2007, vol.35, pp. 516-517.

[9] A. Anders, E.M Oks and G.Yu Yushkov, Production of neutrals and their and effects on the ion charge states in cathodic vacuum arc plasmas, J. Appl. Phys, 2007, vol. 102, pp. 043303/1-7.

[10] A. Anders and G. Yu Yushkov, Puzzling differences in bismuth and lead plasmas: evidence for the significant role of neutrals in cathodic vacuum arcs, Appl. Phys. Lett. 2007, vol. 91, pp. 091502/1-3.

[11] E.M Oks, G.Yu Yushkov and A. Anders, Temporal development of ion beam mean charge state in pulsed vacuum arc ion sources, Rev. Sci. Instrum., 2008, vol. 79, 02B301/1-

E-mails of authors:

oks@opee.hcei.tsc.ru gyushkov@opee.hcei.tsc.ru aanders@1bl.gov 